\title{
DOCENTES UNIVERSITARIOS CON DISCAPACIDAD: EXPERIENCIA Y ENSEÑANZA
}

\author{
Eduardo Enrique Delgado Polo ${ }^{1}$ \\ Universidad Pedagógica Nacional. Bogotá, Colombia
}

\begin{abstract}
RESUMEN
En este artículo se presentan los resultados preliminares de una exploración desarrollada para identificar las formas cómo la experiencia de la discapacidad se refleja en la enseñanza de profesores universitarios con esta condición. Participaron en este estudio seis profesores con discapacidad, actualmente en ejercicio docente en distintas universidades de Bogotá, Colombia. Los resultados parciales que aquí se presentan corresponden al análisis de los registros videográficos, enfatizando en la definición como gesto docente, de acuerdo a la Teoría de la Acción Didáctica Conjunta y la metodología de la Clínica Didáctica para el análisis de situaciones de enseñanzaaprendizaje. Se encontró que la experiencia de la discapacidad se evidencia de forma clara en los gestos definitorios de los profesores y tiene un papel muy importante en los procesos educativos.
\end{abstract}

\section{Palabras clave}

Profesores universitarios con discapacidad, Teoría de la Acción Didáctica Conjunta, experiencia de discapacidad, estilos de enseñanza.

\begin{abstract}
This article presents the preliminary results of a developed scan to identify the ways how the disability experience is reflected in the teaching of university professors with this condition. Six teachers with disabilities, currently in-service teaching at different universities in Bogotá, Colombia participated in this study. The partial results presented here correspond to the analysis of structure of videographic registers, emphasizing the definition as teaching gesture, according to the theory of didactic joint action and the methodology of clinical didactic for the analysis of teaching-learning situations. We found that the experience of disability is evidenced clearly in defining gestures of teachers and has a very important role in the educational process.
\end{abstract}

\section{Keywords}

University professors with disabilities, theory of didactics joint action, experience of disability, teaching styles. 


\section{UNIVERSITY PROFESSROS WITH DISABILITIES: EXPERIENCE AND TEACHING}

Las investigaciones relacionadas con discapacidad tradicionalmente se han centrado sobre todo en aspectos de naturaleza demográfica, generalmente bajo enfoques descriptivos y con objetivos relacionados con la situación de las personas con discapacidad, la prestación de servicios y la calidad y cobertura de los mismos.(OMS, 2011; Cruz, Duarte, Fernández y García, 2013) Actualmente se exploran campos como la participación, la calidad de vida y la inclusión, (Duk, 1999; Schalock \& Verdugo, 2007; Wehmeyer, 2007; Palacios, 2008; Moriña Díez, 2008; Barton, 2011) entre otros, con el objetivo de abrir caminos en dimensiones distintas, comprensivas, que, transiten por perspectivas más sociales y menos reduccionistas. En esto han tenido mucho protagonismo las personas con discapacidad, los colectivos que han conformado y que cada día participan en mayor grado en lo público y lo académico, en todas las áreas del desempeño humano. Las aproximaciones que nos permiten construir conocimiento en discapacidad, desde perspectivas más amplias y participativas, apuntan a considerar caminos y experiencias que contribuyen a mejorar la formación de educadores y a la educación en general. Tal es el impacto de las lecciones aprendidas con la participación de las personas con discapacidad.

La investigación titulada "Docentes universitarios con discapacidad: Experiencia y Enseñanza", se propone como objetivo central la identificación de elementos del perfil docente de profesores con discapacidad que tengan relación con su experiencia personal de la discapacidad y, por esta vía, con su forma de enseñar. Tal aproximación dialoga con los planteamientos teóricos sobre la formación de maestros y perspectivas como la inclusión y la consideración de la diferencia en el acto educativo. A partir de los resultados de este estudio, se esbozarán elementos que podrían considerarse para la construcción de un perfil del "docente inclusivo" y se visibilizará el papel de la experiencia personal en el estilo de enseñanza del docente, como componente fundamental del mismo.

En este trabajo se presentarán los análisis preliminares de la exploración de estos rasgos, centrándonos en el gesto docente denominado definición al interior de la Teoría de la Acción Didáctica Conjunta (TADC), desarrollada por Sensevy (2007) a partir de los planteamientos de Brousseau (1998). Igualmente, la metodología elegida para el análisis acorde con esta teoría es la denominada Clínica Didáctica. (Rickenmann, 2007)

\section{Planteamientos: Teoría de las situaciones didácticas.}

La acción didáctica, acción dirigida y a la vez conjunta por excelencia, se estructura de múltiples formas. Sensevy (2007) propone el juego didáctico como la forma de interacción por excelencia entre los implicados en el proceso de aprendizaje, en el cuál se pueden identificar las características "afectivas, efectivas y pragmáticas" (Sensevy, 2007, p. 7 y ss) de las acciones desarrolladas. Define este juego como "orgánicamente cooperativo" y esta cooperación se basa en la producción y ajuste mutuo de estrategias que permitan la construcción del saber conjuntamente entre profesor y estudiantes. Este juego didáctico juego de saber- tiene características constitutivas muy importantes, -llamados "gestos docentes" por el autor, uno de los cuáles es la "devolución", en la que el estudiante debe "asumir la responsabilidad de jugar verdaderamente el juego". La idea es partir de un problema que el maestro propone y que requiere la producción de estrategias por parte del estudiante. No es un simple proceso de apropiación de saberes o procedimientos sino más bien, de génesis de estrategias para saber o proceder. El maestro produce "sistemas de signos", que propician la generación de estrategias por parte del estudiante. Esto se hace en un "contexto cognitivo común", es decir, por medio de un conjunto de significaciones que se comparten, en las que se introduce el medio -el "juego de saber"-. Sensevy señala que este contexto común es, a la vez, necesario e insuficiente, lo que permite que por medio del juego de saber el medio se convierta en antagónico de este contexto común. Brousseau, (1998, citado en Sensevy, 2007) ejemplifica este juego de saber, cuando el maestro introduce cuestionamientos que interrogan ese contexto común, rompiendo su completitud y mostrando la insuficiencia del contexto para responder todas las preguntas, por lo que se requiere de otro tipo de estrategias producidas por el estudiante. En la situación de clase se desarrollan 
procesos que el maestro moviliza como parte de su enseñanza, para provocar la emergencia de estas estrategias. Lo hace por medio de sus acciones en clase, denominadas en adelante "gestos docentes". Aunque existen muchos, el autor caracteriza cuatro principales. La devolución es uno de estos. Se realiza cuando el maestro transfiere la responsabilidad del desarrollo del tema al estudiante. A través, por ejemplo, de la preparación y presentación de un tema, el estudiante introduce otros aspectos del saber, explora comprensiones y construye propuestas. Asume la responsabilidad-compartida- del desarrollo de la clase. Otros gestos son la regulación y la institucionalización. La regulación es entendida como la intervención que realiza el maestro frente al desarrollo de las actividades, dirigida al mantenimiento de la orientación hacia los objetivos propuestos. La institucionalización se refiere a la contrastación y comprensión a cargo del maestro de los conocimientos construidos en clase, con teorías y marcos generales de interpretación, aceptados por la mayoría de los expertos, permite que se establezcan las cercanías y distancias con estos marcos generales y en qué medida lo que se viene construyendo en clase representa una corroboración o un camino inédito. Un cuarto gesto, la definición, se entiende como el encuadre que el maestro realiza, referido al juego que se juega, las reglas básicas, los contenidos, el contrato didáctico, es decir, el contexto significativo y de acciones pertinente para jugar el juego. En general, la definición se realiza al inicio del ciclo y clase. Este gesto, es uno de los más complejos y más ricos, con múltiples formas de expresarse, por lo que se ampliará su significado: De acuerdo con Sensevy (2007), "cuando hablamos de «definición del juego», nos referimos a la transmisión de reglas constitutivas, definitorias del mismo..." los alumnos deben comprender, por lo menos en el primer nivel del juego efectivo, qué es lo que están jugando. El gesto de definición también se expresa en la postura del maestro, en lo que considera importante o pertinente como claves para ganar el juego.

Esta acción docente, así como las otras tres mencionadas, permite comprender cómo se construye el saber y cómo se evidencian los juegos de aprendizaje, desde lo que se denomina "hacer jugar el juego", como un conjunto de descriptores que evidencian estas dinámicas.

\section{La discapacidad en el contexto del juego de saber}

Ahora bien, atendiendo los objetivos de la presente investigación, es posible hipotetizar una conexión entre los gestos docentes y la experiencia propia del profesor con la discapacidad. Esta experiencia está presente en muchos de estos. Es importante hacer la aclaración sobre cómo se entiende la discapacidad: Siguiendo a Schalock (1999), la discapacidad se origina en la interacción del sujeto con el ambiente, interacción que resulta agresiva o a pérdida cuando las barreras (actitudinales o físicas) que presenta este ambiente son obstaculizantes para el desempeño del sujeto por sus condiciones propias de competencia o pensamiento. Esta concepción de discapacidad toma distancia del enfoque médico funcionalista, que se centra en la alteración del funcionamiento del sujeto asociándola a enfermedad. Percibir de otras formas, como sucede con los sordos o los ciegos, moverse de manera diferente o en ruedas, hablar siguiendo otras prosodias, forman parte de la experiencia a lo largo de toda la vida en donde ésta relación con el ambiente ha tenido que replantearse frecuentemente y así como en los demás ámbitos cotidianos, para los profesores con discapacidad constituyen rasgos que se manifiestan en acciones docentes determinadas, En todos los casos, contribuyen a las formas como el gesto de "definición" se manifiesta, por cuanto son rasgos que impregnan de manera clara el encuadre de los juegos de saber.

\section{Metodología}

Para el presente estudio, se siguieron tres vías de exploración: la Clínica Didáctica, (para la cual se desarrollaron videograbaciones de tres clases simultáneas) construcción de Relatos de Experiencia y aplicación del Inventario de Estilos de Enseñanza. En este artículo se presentan los resultados parciales de la primera vía.

Clínica Didáctica. De acuerdo con Rickenmann, y Juanola, (2012) se sitúa el inicio de esta perspectiva clínica sobre las didácticas, en la escuela francesa aproximadamente en 1980; muy reciente si se toma en cuenta la tradición mucho más desarrollada de la producción investigativa en el campo de las 
disciplinas escolares (Brousseau, 1991; Chevallard, 1997) y de la lengua (Bronckart \& Schneuwly, 2004). Más recientemente, este marco metodológico se ha incorporado tanto en la investigación como en la formación de otras disciplinas escolares como la educación física (Amade-Escot, 2007) y las enseñanzas artística y musical (Rickenmann et al., 2009 Juanola y Rickenmann, 2012).

Su principal característica es que se centra en la "observación, descripción y comprensión de las situaciones didácticas reales" (Rickenmann, 2012) con el fin de comprender cómo se da su funcionamiento. Este no se visualiza automáticamente, sino que requiere de cuestionamientos previos sobre los saberes que se enseñan y las prácticas que de estos se desprenden. Esto es especialmente relevante frente a concepciones como la discapacidad y las prácticas que de estas se han derivado. Se proponen entonces, los "observables pertinentes" (Rickenmann, 2012). Que, al igual que en la clínica médica, se reconocen dentro de un marco general explicativo el cual, a la vez es interrogado por los nuevos hallazgos.

\section{Los profesores participantes}

La configuración del grupo de profesores participantes se dio como resultado de unas búsquedas en diversas universidades de Bogotá, tanto públicas como privadas en donde se ubicaron profesionales con discapacidad desempeñándose como profesores. Además de lo anterior, se estableció como condición que la discapacidad estuviera presente desde edades muy tempranas, formando parte de la experiencia central a lo largo del ciclo vital de la persona. Se contactó a los profesores, se realizó una entrevista inicial en la que se presentó la investigación, sus objetivos y propósitos y se estableció la viabilidad de la participación de acuerdo a horarios e interés propio. Se conformó un grupo de seis profesores, (cuatro hombres y dos mujeres) en cuatro universidades. Dos de ellos, sordos, uno ciego, uno con secuelas de lesión neurológica temprana - Parálisis cerebral- y dos con alteraciones de movilidad secundarias a trauma severo en primeras etapas de vida. Todos ellos, profesores activos, con interés en participar del estudio.

\section{Categorías del análisis}

La observación de las formas como se evidencia la experiencia de la discapacidad en el gesto denominado definición, se describe a continuación. Estas evidencias se recogieron en los protocolos, que contienen la descripción de la observación de las clases de los maestros participantes. Se tuvieron en cuenta aspectos como los momentos en los que se desarrolla la clase, acuerdos de trabajo entre profesores y estudiantes, asignación de responsabilidades y roles, conducción de los debates, etc., entre otros, en los que se buscaron las expresiones a través de las cuáles se hace evidente la experiencia con la discapacidad por parte de los maestros participantes. Esto se hizo teniendo en cuenta específicamente el gesto docente denominado definición.

Es importante señalar que las evidencias observables son relativas a la experiencia de la discapacidad, más que a la discapacidad misma. Es decir, que lo que se observa no es la evidencia de la alteración o la dificultad, -la sordera, la ceguera o la parálisis cerebral, por ejemplo- sino cómo esta se ha convertido en una fuente de experiencia que aporta también formas de ser y estar en el mundo, también en rasgos presentes en la enseñanza.

La experiencia, como acontecimiento profundo y resultado, como irrupción de lo que es nuevo cambiando el orden de lo conocido y reclamando la búsqueda de sentido para lo que no lo tenía o no se le había dado, completamente propia e implicadora, personal, (Dewey, 1995; Gadamer, 1997; citados en Contreras y Pérez de Lara, 2010) es la clave de interpretación en la que se ha centrado todo el análisis. Ésta, que contiene el saber acumulado de toda la existencia personal, que resulta de los acontecimientos que le suceden cotidiana y permanentemente a cada persona, se accede en esta pesquisa, desde un lugar de enunciación, de sentidos construidos y por construir, denominado "discapacidad". A partir de situarse en este lugar (sedimentado en algunos aspectos, difuso en muchos otros) los participantes y el investigador se han encontrado interrogados por sí mismos y por los otros, preguntándose por el saber de esas experiencias. Así, la búsqueda de evidencias sobre cómo la experiencia de la discapacidad se muestra en la enseñanza, se vuelve la indagación de rasgos, de indicios sobre cómo este saber se evidencia en 
comportamientos específicamente ligados al proceso de la enseñanza. En este sentido, muchos rasgos emergen como posibilidades, de los cuales fue necesario elegir aquellos que, en la visión del investigador, expresan de manera más completa ese saber sedimentado, elaborado, construido a lo largo de toda la vida viviendo con discapacidad.

Los parámetros (acciones o comportamientos observables, en el gesto de definición, que evidencian la experiencia de la discapacidad) que emergieron del análisis de la observación contenida en los protocolos fueron:

1. Referencias a sí mismo y a su propia experiencia para ejemplificar alguna situación o argumento. Se refiere a expresiones que el profesor usa dentro de sus clases, en las que hace expresa su experiencia con la discapacidad y su conocimiento del ámbito significativo que esta representa; estas expresiones tienden a reforzar su autoridad frente al tema.

2. Demostraciones sobre el uso de ayudas o herramientas específicas. Hace referencia a conductas realizadas por el profesor en donde demuestra cómo se usa una herramienta o ayuda relacionada con la discapacidad. Entran en esta categoría los sistemas de comunicación aumentativa o alternativa, la lengua de señas, el uso de prótesis o sillas de ruedas.

3. Adaptación del entorno por su condición particular. Esta categoría contempla los ajustes que el profesor realiza en el entorno teniendo en cuenta condiciones personales como movilidad reducida, sordera, ceguera o necesidades de accesibilidad.

4. Alusión a colectivos, experiencias institucionales o grupos a los que se pertenece como respaldo a su experiencia con la discapacidad. Esta categoría implica las referencias expresas por parte del profesor a la pertenencia presente o pasada a colectivos de personas con discapacidad o asociaciones que trabajan con el tema, así como a proyectos o iniciativas que actúan en la búsqueda de transformaciones de las comprensiones sociales frente a la discapacidad.

5. Acompañamiento al proceso formativo del estudiante. Se refiere a las conductas que el profesor expresamente desarrolla para acompañar el trabajo de los estudiantes, de manera individualizada o en pequeños grupos. Incluye la realización de tutorías, la retroalimentación directa y la revisión del proceso a lo largo del curso. Esta categoría, de manera más amplia que las anteriores, se refiere al gesto denominado definición y emerge de manera fuerte en el análisis como evidencia de la experiencia con la discapacidad, ya que los profesores participantes se muestran especialmente orientados a actuar en esta dirección, muy posiblemente por la importancia crítica que este aspecto ha tenido en su propia experiencia de vida. Igualmente, resulta más relevante en el contexto universitario, en donde este acompañamiento no se considera importante en general.

Las categorías anteriores son gestos fundamentalmente definitorios, aunque su relación con otros gestos docentes (como la regulación o la devolución) es dinámica y muestra la interactividad característica de los juegos de saber; en este sentido, contribuyen a caracterizar estos otros gestos.

\section{Análisis Transversal. Lo común y lo distinto.}

\section{Resultados}

Desde el análisis de la relación que explora este estudio entre la experiencia de la discapacidad y

la forma de enseñanza, evidenciada en el gesto docente denominado definición, se plantean los siguientes contrastes y aspectos comunes:

Frente a los parámetros sugeridos, el primero, referirse a sí mismo o a su propia experiencia con la discapacidad como ejemplo, cuatro de los seis profesores participantes lo hacen, en mayor o menor medida.

El segundo parámetro, hacer demostraciones, se hace más evidente en los seis profesores. En mayor grado, el profesor $\mathrm{H}$ evidencia a través de este recurso su experiencia y conocimiento de la discapacidad, cuando guía sus clases a través de la forma en cómo se usa la plantilla para el braille; Los profesores $\mathrm{N}$ y G, lo hacen en menor medida, -más en relación con el parámetro siguiente, la adaptación 
del medio - pero está presente en sus clases. De todos modos, en el caso de estos profesores, estas adaptaciones contribuyen a definir las formas de relación y comunicación, así como lo que los estudiantes pueden esperar en lo que respecta al tipo de relación.

El tercer parámetro, adaptar el entorno a su condición propia, es evidente en los seis profesores. En el caso de los profesores $\mathrm{O}$ y $\mathrm{J}$, es donde se muestra más claramente, debido a que la comunicación requiere de manera obligatoria de la intérprete, lo cual da una forma característica a la clase. La forma de la interpretación, el lugar en donde se sitúa el intérprete y su propia dinámica, afectan también. Los profesores $\mathrm{H}, \mathrm{N}$ y $\mathrm{G}$, adaptan el entorno, aunque de manera menos rigurosa. La profesora $\mathrm{R}$ no realiza adaptaciones vinculadas a su condición personal, sino, ella se adapta al entorno.

El cuarto parámetro, referirse a colectivos, experiencias institucionales y/o a imaginarios sociales, se muestra evidente en cuatro de los seis profesores participantes. Los profesores $\mathrm{H}, \mathrm{O}, \mathrm{N}$ y $\mathrm{G}$, hacen estas referencias expresas. Los profesores $\mathbf{J}$ y $\mathbf{R}$, no las utilizan relacionadas con discapacidad, sino con los aspectos generales del contexto significativo de sus respectivas clases.

Con relación al quinto parámetro, acompañamiento del proceso formativo del estudiante, se encuentra que los seis profesores lo realizan. Este acompañamiento al desempeño individual del estudiante se muestra como un grado de cercanía al proceso, con un seguimiento permanente de lo que sucede con el estudiante frente a su formación; no solo frente a la construcción del conocimiento o la adquisición de habilidades, sino frente a la consideración del sujeto particular que se está formando. Este aspecto, eminentemente pedagógico, se interpreta desde la experiencia que estos profesores han tenido con la discapacidad, que les ha aportado, entre otras cosas, una mayor sensibilidad a los procesos. Este seguimiento es propio de la enseñanza primaria y básica, reduciéndose hacia la media y la superior, en donde se centra en el cumplimiento de responsabilidades específicas, entrega de productos y resolución de pruebas. En donde se conserva de forma parecida a lo evidenciado aquí, es en las prácticas y los ciclos formativos que requieren de desarrollos procedimentales. Es interesante que los profesores participantes, en sus seminarios incluyan y desarrollen este aspecto. A continuación, se presenta una tabla que esquematiza lo anterior.

Tabla 1

Resumen de las evidencias de la experiencia de la discapacidad en la "definición" docente

\begin{tabular}{|c|c|c|c|c|c|c|}
\hline \multirow[t]{2}{*}{ Parámetro } & \multicolumn{6}{|c|}{ PROFESORES } \\
\hline & $\mathrm{H}$ & $\mathrm{O}$ & $\mathrm{J}$ & $\mathrm{N}$ & $\mathrm{G}$ & $\mathrm{R}$ \\
\hline $\begin{array}{l}\text { Referencia a sí } \\
\text { mismo }\end{array}$ & $\sqrt{ }$ & $\Leftrightarrow$ & $\sqrt{ }$ & $\sqrt{ }$ & $\sqrt{ }$ & 1 \\
\hline Demostraciones & $\sqrt{ }$ & $\sqrt{ }$ & $\sqrt{ }$ & $\sqrt{ }$ & $\Leftrightarrow$ & $\sqrt{ }$ \\
\hline Adaptar el entorno & $\sqrt{ }$ & $\sqrt{ }$ & $\sqrt{ }$ & $\sqrt{ }$ & $\sqrt{ }$ & $\sqrt{ }$ \\
\hline $\begin{array}{l}\text { Referirse a } \\
\text { colectivos }\end{array}$ & $\sqrt{ }$ & $\sqrt{ }$ & 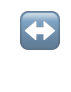 & $\sqrt{ }$ & $\sqrt{ }$ & / \\
\hline $\begin{array}{l}\text { Acompañamiento } \\
\text { al proceso } \\
\text { formativo del } \\
\text { estudiante }\end{array}$ & $\sqrt{ }$ & $\sqrt{ }$ & $\Theta$ & $\sqrt{ }$ & $\sqrt{ }$ & $\sqrt{ }$ \\
\hline
\end{tabular}

$$
\sqrt{ } \text { : Presente. } A: \text { parcialmente presente } \quad \text { : No presente. }
$$

\section{Algunas conclusiones}

Teniendo en cuenta que los profesores participantes fueron seleccionados bajo dos criterios fundamentales: que tuviesen discapacidad desde muy temprana edad y que actualmente se desempeñaran como profesores en alguna universidad, emergieron unas subagrupaciones que permitieron desarrollar núcleos de Análisis: Relación de la experiencia de la discapacidad con la forma de enseñar (comprendiendo el tipo de interacción con el medio y los gestos docentes) y con los contenidos enseñados 
y la relación entre la experiencia de la discapacidad y las competencias para enseñar. Tres profesores enseñan clases directamente relacionadas con discapacidad como contenido central y tres enseñan otras

materias; tres de ellos tienen formas de comunicación distintas a las de otros profesores (Lengua de señas -intérprete y braille).

En lo relacionado con las secuencias didácticas que desarrollan estos profesores, se advierte que estas tienen una estructura similar entre sí, mostrándose como la típica de la docencia universitaria; (presentación general por parte del profesor, asignación de responsabilidades a los estudiantes, presentaciones de éstos, debate, conclusiones) sin embargo, en todos los casos, el componente del debate es usado para hacer avanzar el conocimiento y construir alternativas de comprensión. En los casos de los profesores $\mathrm{H}$ y $\mathrm{O}$, además de la secuencia descrita, se incluyen ejercicios prácticos que son retroalimentados y ajustados permanente e inmediatamente. De todos modos, las presentaciones de los estudiantes, en todos los casos funcionan de esta manera. La secuencia didáctica desarrollada busca que los estudiantes participen y propongan desde un rol activo, planteando diferentes caminos a la interpretación; las clases se realizan en un clima de "taller", invitando a la construcción. Teniendo en cuenta lo anterior, pueden caracterizarse dos formas de los saberes que se enseñan y que forman parte de la estructura de la secuencia didáctica: en primer lugar, el saber disciplinar, que se expresa concretamente al interior de la clase o seminario y que le constituye. Es el tema general sobre el que trata la clase.

La segunda forma de saber que se enseña, combina el saber procedimental con el saber experiencial, constituyéndose en una perspectiva compleja sobre la experiencia de la discapacidad. Este saber, como resultado de la elaboración de la vivencia, está profundamente arraigado en la subjetividad y la constituye.

Con relación a lo anterior, se advierte que los profesores participantes trabajan mucho sobre la discusión y el debate como procesos dinámicos de construcción de sentido en las secuencias didácticas que desarrollan: se "apuestan" en el terreno que puede ser lugar de encuentro con sus estudiantes: La vivencia. Es un proceso con muchos componentes del aprendizaje situado, en donde "se aboga por una enseñanza centrada en prácticas educativas auténticas, las cuales requieren ser coherentes, significativas y propositivas" (Díaz -Barriga, 2003) y que son, fundamentalmente, prácticas cotidianas.

Esta es una característica común a todos los profesores con quienes se trabajó y representa un valor importante en la formación de profesores. Una vez más aparece como un gran valor la experiencia del profesor, más que el conocimiento de unas teorías o unas técnicas. Ese es el componente fundamental, lo que lo hace maestro. (En algún sentido, era lo que se quería en el pasado con la vinculación de los “expertos" a las universidades en la forma de catedráticos) Es, en últimas, la mejor acepción de la frase: "ese maestro sí sabe". Lo anterior se da al interior de unos juegos de aprendizaje en los que el profesor combina la definición, la devolución, la regulación y la institucionalización siguiendo el proceso que van desarrollando los estudiantes, siempre en zona de desarrollo próximo. La experiencia vital con la discapacidad a lo largo de la vida configura formas propias de percibir, ser y actuar en el mundo. Más allá de lo evidente funcional, esta experiencia comporta la vivencia intersubjetiva, la interacción, inédita muchas veces, con un mundo que tiene rutas y ritmos normalizados. Es la experiencia del desafío, de la pregunta y la búsqueda constante, de la revisión de los pasos y la reorganización de la esperanza. Es un saber poco conocido y muchas veces silenciado por el saber colonial médico, que subordina la experiencia a nivel de obstáculo. Por el contrario, la experiencia es la base del saber, es la vivencia comprendida y contrastada, preguntada mil veces y reconfigurada cada día. La experiencia con la discapacidad es ordenadora de la subjetividad.

En asignaturas relacionadas directamente con discapacidad, estos procesos se hacen más fácilmente vinculables y se leen casi que directamente; en otro tipo de asignaturas, sin embargo, se evidencia que el maestro ha desarrollado unos "rasgos pedagógicos" en su quehacer que podrían ser atribuibles a su experiencia con la discapacidad, que le permiten formas de aproximación, estilos de relación más abiertos y provocadores en lo que atañe a la interacción comunicativa. Más que querer que 
los estudiantes se aprendan unas teorías, se busca construir con el estudiante comprensiones contextualizadas en torno a las mismas y desarrollar habilidades de pensamiento vinculadas con los procesos, más que con los conceptos. De todos modos, podría afirmarse que en las clases que trabajan centralmente con conceptos científicos, por ejemplo, el lugar de la teoría es más importante que el de la experiencia, aunque la experiencia les aporta las formas de enseñar.

En resumen, las competencias profesorales (Enseñar, Formar y Evaluar) se desarrollan de manera importante en relación con la experiencia con la discapacidad; como se hace evidente en el acompañamiento que realizan los profesores a los procesos de los estudiantes, la experiencia con la discapacidad durante toda una vida, que les implica, entre otras cosas, colocar especial atención a los pasos que dan, a la forma de hacer las cosas, a la comunicación, a las relaciones con los demás, etc., les aporta un proceder con estos rasgos propios, como parte de su estilo docente. 


\section{Referencias}

Abello D. y Hernández C. (2012). Inventario de Estilos de Enseñanza. Bogotá: UPN.

Ainscow, M. y Booth, T. (2000). Index for Inclusion. Centre for Studies on Inclusive Education (CSIE), Bristol UK.

Barton, L. (2011). La investigación en la educación inclusiva y la difusión de la investigación sobre discapacidad. Revista Interuniversitaria de Formación de Profesorado, vol. 25, núm. 1, abril, 2011, pp. 63-76.

Brogna, P. (2008). Visiones y Revisiones de la Discapacidad. Buenos Aires: Cinca.

Brousseau, G (1990 y 1991). ¿Qué pueden aportar a los enseñantes los diferentes enfoques de la didáctica de las Matemáticas? (Primera parte) En Enseñanza de las ciencias, Vol. 8, № 3, p. 259-267, (Segunda parte) En Enseñanza de las ciencias, ISSN 0212-4521, Vol. 9, No 1, p. 10-21.

Brousseau, G. (2007). Iniciación al estudio de la teoría de las situaciones didácticas. 1 ed. Buenos Aires: Libros del Zorzal.

Chevallard, Y. (1997). La transposición didáctica. Del Saber Sabio al Saber Enseñado. Buenos Aires: AIQUE.

Contreras, J., Pérez de Lara, N. (Eds.). (2010). Investigar la experiencia educativa. Madrid: Morata.

Cruz I, Duarte C, Fernández A, García S. (2013.) Caracterización de investigaciones en discapacidad en Colombia 2005-2012.Revista de la Facultad de Medicina, Universidad Nacional. 2013 Vol. 61 No. 2: 101-109.

Juanola R., Rickenmann R, (eds.) (2012). Diálogos sobre investigación de la acción didáctica conjunta: retos y perspectivas. Capítulo II. En: Coll Narratives d'art i educació;. Girona: Documenta Universitaria.

Moriña, A.,Vulnerables al silencio: historias escolares de jóvenes con discapacidad.Revista de Educación, 353. Septiembre-Diciembre 2010, pp. 667-690.

Organización Mundial de la Salud y Banco Mundial. (2011). Informe mundial sobre la discapacidad. Recuperado de: http://new.paho.org/arg/images/Gallery/Informe_spa.pdf.

Palacios, A. (2008). El modelo social de discapacidad: orígenes, caracterización y plasmación en la Convención Internacional sobre los Derechos de las Personas con Discapacidad. Madrid: CINCA.

Pérez de Lara, N. (1998) La capacidad de ser sujeto. Barcelona: Laertes.

Rickenmann, R. (2007). El rol de los artefactos culturales en la estructuración y gestión de las secuencias de enseñanza-aprendizaje. Recuperado de: www.unige.ch/fapse/clidi/textos/artefactosculturales-RR.pdf

Rickenmann, R. (2007.) Metodologías clínicas de investigación en didácticas y formación del profesorado: un estudio de los dispositivos de formación en alternancia. Universidad de Ginebra (Suiza)

Schalock, L. R. \& Verdugo, Alonso M. A. (2007). El concepto de calidad de vida en los servicios y apoyos para personas con discapacidad intelectual. Revista de Educación en humanidades, 38 (4) (224), 21-36.

Schalock, R.L. (1999). Hacia una nueva concepción de la discapacidad. Siglo Cero, volumen 30, No. 181, $5-17$.

Sensevy, G (2007). Categorías para describir y comprender la acción didáctica. Traducción de Juan Duque y revisión de René Rickenmann. 5-34. En Sensevy, G \& A. Mercier Agir ensemble: l'action didactique conjointe du professeur et des élèves. Rennes: PUR.

Vygotski, L.-S. (2001). Pensamiento y Lenguaje; Conferencias sobre psicología, en Obras escogidas. Tomo II. Madrid: Antonio Machado Libros.

Wehmeyer, M. (2007) Theory in self - determination: foundations for educational practice. Sage Pub. 